\title{
Reflecting on the achievements and looking to the future of an established rheumatology journal
}

\author{
Armen Yuri Gasparyan ${ }^{1}$ (D)
}

Published online: 25 September 2017

(c) Springer-Verlag GmbH Germany 2017

Rheumatology is one of the rapidly developing clinical disciplines, which is actively contributing to interdisciplinary research. The innovation and knowledge accumulation in this field may impress any specialists, who encounter patients with rheumatic and musculoskeletal disorders. Modern rheumatologists are privileged since they are supplied with regularly updated clinical recommendations, reflecting the enhanced armamentarium for diagnosis and management of rheumatic diseases. All these recommendations result from concerted action of expert rheumatologists, who systematically process evidence-based data, take advice of allied specialists, analyze patient responses, and target highly cited and widely promoted rheumatology journals [1]. The scientific prestige of these journals, which is partly due to the endorsements of professional societies and high number and 'weight' of citations, attracts elite authors and reviewers. Consequently, the best quality research, reviews, and clinical recommendations often appear in the same journals. Top rheumatology journals with their exemplary online platforms, regularly updated instructions, publishing strategies, and format of articles are models for lowerrank indexed and emerging periodicals in the same subject category.

As a rule, researchers involved in clinical trials and large cohort studies on rheumatic diseases choose top rheumatology journals as prime targets for their research output and work their way down the 'impact factor ladder'. Such an

Armen Yuri Gasparyan

a.gasparyan@gmail.com

1 Departments of Rheumatology and Research and Development, Dudley Group NHS Foundation Trust (Teaching Trust of the University of Birmingham, UK), Russells Hall Hospital, Dudley, West Midlands DY1 2HQ, UK approach takes a lot of time and efforts of authors, reviewers, and editors since there are only a few top-impact rheumatology journals and their publishing capacities are not unlimited. Rejections of great articles by these journals, which are upsetting for all, call for more weighted selection of the target journals. There are many established rheumatology journals with reasonably high citation metrics, such as Rheumatology International, which can serve as prime targets for evidence-based and ethically sound articles.

Launched in 1981, Rheumatology International has matured over time, established and maintained its ties with professional teams from all over the world, set new ambitious goals, and positioned itself as a truly international reference for topics rarely covered elsewhere [2]. The Journal gradually increased its annual publication rates and accommodated numerous randomized controlled trials [3]. Over the past few years, the expansion of the reviewers' pool has been remarkably successful. The best reviewers' professional contribution and adherence to research reporting standards, deserving credit, international recognition and editorial promotion, allowed filtering out most submissions with poor structure, methodological flaws, irreproducible results, and redundant conclusions. Along with the impressive achievements and despite the availability of powerful pre-publication validation tools, the journal editors have also confronted the issue of retractions (https://doi.org/10.1007/ s00296-016-3471-x). Although retractions are uncommon across indexed rheumatology journals [4], even a single 'self-cleaning' act calls our attention to the fact that any great journal can be affected by short-sighted and apparently unethical attitude of some authors. Our reviewers and editors are, therefore, encouraged to take a more proactive stance and report any instance of unethical copying, conflicting and erroneous reporting, lack of transparency, and other forms of misconduct in the submissions to the journal. 
In the wake of the move toward open access, many online journals have been launched to cascade and accommodate the growing number of articles rejected by established rheumatology and allied periodicals. Some of these journals, supported and guided by professional societies, have attracted good articles and ethical citations and got indexed by prestigious databases, such as Scopus, Web of Science, and MEDLINE. But many other start-up journals have failed to meet high publishing standards and embarked on a wide range of unethical practices, damaging reputation and wasting their authors' efforts [5, 6]. Choosing titles mimicking established ones has been recently exemplified as one of the appalling tricks of bogus rheumatology journals [7]. Any such move aims at misleading novice authors, and particularly busy clinicians, who rarely check the list of indexed journals and overlook the importance of carefully evaluating the target journal profile.

Established rheumatology journals, including Rheumatology International, can take the lead by setting publishing standards in the field, attracting the best authors, and improving their contributors' science communication skills. Editors are reminded that the most important and enduring role scholarly journals play is sharing discoveries and knowledge among professionals [8]. The best rheumatology research should be represented by evidence-based, widely visible, and well cited media to pursue its ultimate goal, which is the improved management of patients with rheumatic and musculoskeletal disorders. At the same time, an established rheumatology journal should not absolutize 'citable' items and should focus more on educational needs of its readers and publish more reviews and innovative opinion pieces, which are entirely abandoned by most start-up journals. Qualitative studies of nurse-led clinics, rheumatic patients' experiences, and emerging non-drug therapies, which rarely find their way to pharma-sponsored media, can be also actively solicited and promoted by the journal editors. Such topics, which are unattractive citation-wise, enlighten new directions of the quality care and relations between patients and professionals $[9,10]$. Healthy mix of various topics and types of articles makes the journal issue readable and attractive for researchers and clinicians.

The 'deluge of information' and sophistication of knowledge transfer in our times necessitate embracing a systematic approach to research and relying more on digital tools. Authors and editors alike are nowadays offered advanced bibliographic searches through which connections are established between known facts and genuinely innovative data. Systematic search strategies, employed by the journal contributors, can make a big difference by providing the readers with properly validated articles.

Editors of this and other established journals often take for granted the availability of editorial management platforms. Such platforms, which are equipped with cross-links to bibliographic databases and contributor identification platforms, facilitate the selection of truly innovative and ethically sound submissions, let alone the acceleration of their transfer to the public domain. By employing full potential of the editorial management system, it is possible not just to streamline processing of submissions, but also ranking of reviewers.

The digitization of the publishing enterprise, among many other benefits, offers enormous opportunities for clinical disciplines heavily relying on audio and visual materials. Rheumatology researchers and authors are now offered an opportunity to enhance their traditional articles with properly edited video clips, depicting locomotor disorders and imaging procedures, which are difficult to describe verbally. Additionally, editors can boost their journal influence by publishing short video articles and interviews with accompanying succinct texts, primarily appealing to patients and busy clinicians [11].

To sum up, the changing publishing landscape offers fascinating opportunities for the growth of this promising rheumatology journal. It has to make an impact by enhancing its traditional values and filling gaps in the field. The internationalization, prioritization of evidence-based data, and enforcement of ethical norms will continue to determine our editorial policies and scope of interests for years to come.

\section{References}

1. Gasparyan AY, Kitas GD (2016) Indexing rheumatology journals: filling gaps in the publishing landscape. Mediterr J Rheumatol 27(1):20-25

2. Carmona L (2013) A full new enthusiastic team. Rheumatol Int 33(4):825-826

3. Lee JW, Chung JH, Jo JK, Lee SW (2014) Analysis of randomized controlled trials in Rheumatology International from 1981 to 2012: methodological assessment. Rheumatol Int 34(9):1187-1193

4. Gasparyan AY, Ayvazyan L, Akazhanov NA, Kitas GD (2014) Self-correction in biomedical publications and the scientific impact. Croat Med J 55(1):61-72

5. Beall J (2017) Predatory journals threaten the quality of published medical research. J Orthop Sports Phys Ther 47(1):3-5

6. Manca A, Martinez G, Cugusi L, Dragone D, Mercuro G, Deriu F (2017) Predatory open access in rehabilitation. Arch Phys Med Rehabil 98(5):1051-1056

7. Publisher backs down in dispute with society over journal name. http://retractionwatch.com/2017/05/04/publisher-backs-disputesociety-journal-name/. Accessed 5 Sept 2017.

8. Fiala C, Diamandis EP (2017) The emerging landscape of scientific publishing. Clin Biochem 50(12):651-655

9. Berry D, Bradlow A, Courtenay M (2008) Patients' attitudes towards, and information needs in relation to, nurse prescribing in rheumatology. J Clin Nurs 17(2):266-273

10. Larsson I, Bergman S, Fridlund B, Arvidsson B (2012) Patients' experiences of a nurse-led rheumatology clinic in Sweden: a qualitative study. Nurs Health Sci 14(4):501-507

11. Bischoff K, Anderson W, Pantilat SZ (2017) A video is worth a thousand words. J Hosp Med 12(9):773-774 